\title{
Écoulement et capture d'aérosols dans les masques respiratoires
}

Camille Duprat (camille.duprat@ladhyx.polytechnique.fr)

Laboratoire d'hydrodynamique de l'École polytechnique (UMR 7646 CNRS)

LadHyX, École polytechnique, 91128 Palaiseau Cedex

\section{Une stratégie essentielle}

dans la lutte contre la pandémie

de Covid-19 est le port

de masques composés de fibres

enchevêtrées non tissées,

qui capturent les gouttes chargées

de virus émises par le porteur

du masque ou présentes

dans l'air ambiant.

Deux mécanismes principaux

de capture des gouttes sont

observés : l'interception directe

de grosses gouttes qui ne sont

capturées qu'en dessous

d'une vitesse critique,

et la capture par impact inertiel

de petites gouttes qui augmente

avec la vitesse des gouttes.

Il est nécessaire de mieux

quantifier ces phénomènes

en fonction de la microstructure

du matériau filtrant pour optimiser

l'efficacité des masques selon

les usages.
Il est désormais établi qu'un vecteur important de transmission de la Covid-19, et d'autres maladies respiratoires, se fait par la respiration de gouttelettes chargées de virus émises par un individu porteur du virus. Lors de la respiration, ces gouttelettes sont de petite taille, généralement inférieure au micron, portées par notre souffle à une vitesse de l'ordre du mètre par seconde. Leur taille augmente lorsque nous parlons, toussons ou éternuons, pour atteindre quelques microns, voire centaines de microns, lors d'un éternuement (fig. 1a et c), avec de fortes variations selon les individus ; les vitesses d'écoulement sont alors plus importantes, de l'ordre de quelques mètres par seconde. Selon leur taille et leur vitesse, ces gouttes vont être transportées par l'écoulement ambiant ou projetées sur des surfaces ou des individus ; elles peuvent parcourir plusieurs mètres avant de s'évaporer, de se déposer sur un objet ou de tomber sur le sol.

Outre la distanciation physique et l'aération des espaces confinés [1], un élément essentiel pour éviter la propagation de cet aérosol de gouttelettes est le port d'un masque. Un masque se doit d'être respirable, c'est-à-dire de laisser le passage de l'air sans avoir à appliquer une dépression trop importante, tout en capturant les gouttes émises par le porteur du masque lorsqu'il expire et celles présentes dans l'air ambiant lorsqu'il inspire. Ce compromis entre respirabilité et efficacité de filtration a conduit à utiliser des textiles, dès l'Empire romain. Après un premier brevet déposé en 1849 pour un filtre de laine, de nombreuses études se sont intéressées à la filtration par des milieux fibreux, avec un essor important dans les années 1930, puis un développement de modèles théoriques au sortir de la Seconde Guerre mondiale, notamment par Irving Langmuir et Katherine Blodgett [2-3]. Aujourd'hui, l'usage de matériaux fibreux pour la filtration d'aérosols est généralisée : composés de nombreuses fibres fines (typiquement une dizaine de microns de diamètre), ils permettent une bonne efficacité de capture (fig. $1 \mathrm{~b}$ et $\mathrm{d}$ ) tout en restant très poreux et perméables (ou respirables). Ces filtres sont généralement composés de fibres non tissées (on parle de "textile non tissé »), c'est-à-dire enchevêtrées et dont la cohésion est assurée par friction ou par adhésion au moyen d'un collage thermique ou chimique. Au LadHyX, nous travaillons depuis plusieurs années sur les interactions entre gouttes et fibres, en particulier dans le contexte de la récupération d'eau douce à partir de brouillard ; nous étudions ainsi la collecte de gouttelettes d'aérosols à l'aide de filets textiles flexibles. Cette question de la filtration de gouttelettes par des fibres a pris un nouveau tournant au début de la pandémie de Covid-19.

\section{Principe de la filtration}

Lorsque l'on envoie un aérosol liquide sur un filtre constitué de fibres, une partie des gouttelettes contenues dans l'aérosol est capturée par les fibres, puis elles s'y accumulent et y coalescent; c'est le principe de la filtration au moyen des filtres dits à coalescence [4]. La filtration est généralement caractérisée par deux grandeurs : la perte de charge, c'est-à-dire la différence de pression entre l'amont et l'aval du filtre, 


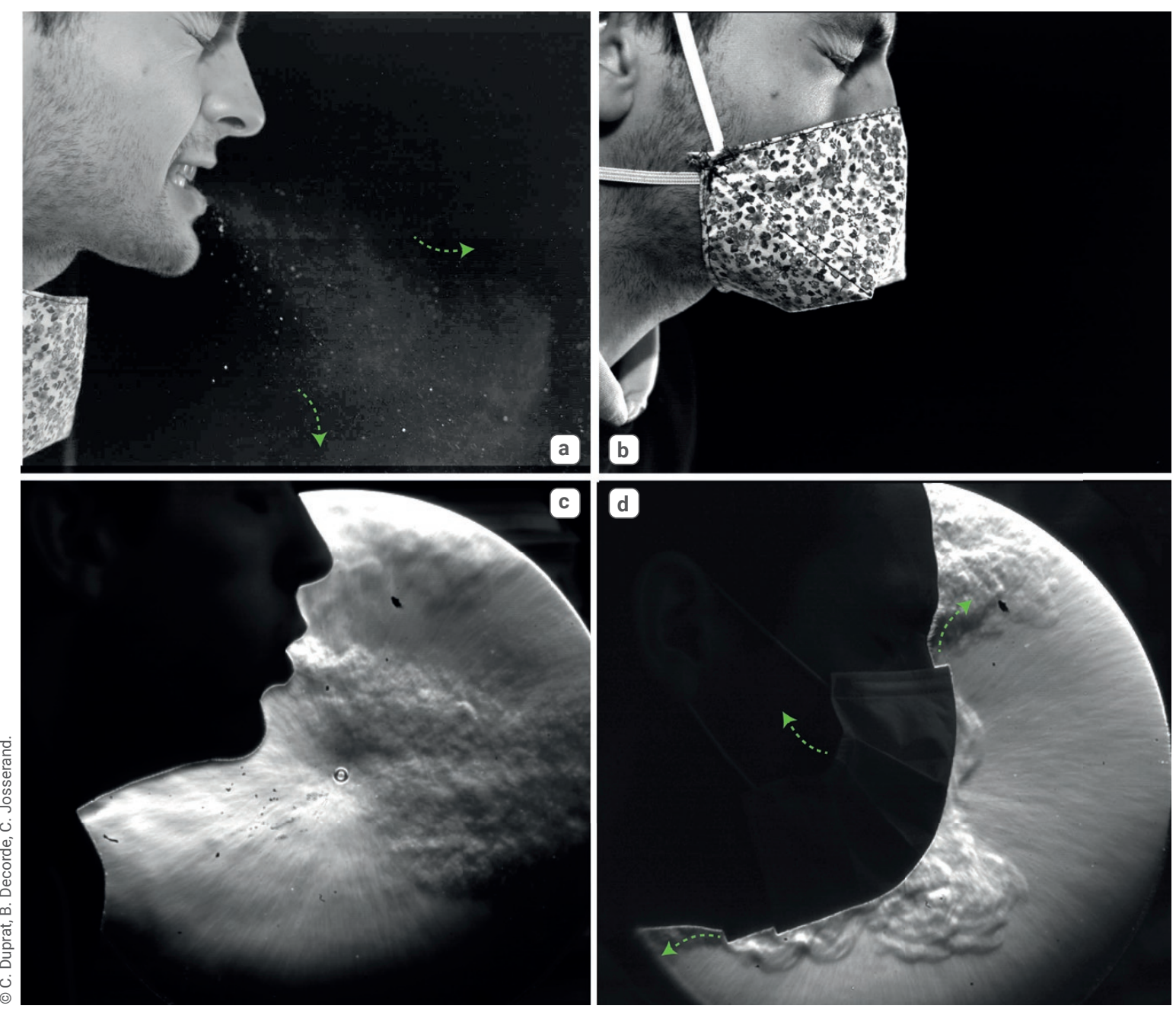

1. Visualisation des aérosols générés lors de la toux ou des éternuements.

(a-b) Images prises lors d'un éternuement, sans masque (a) et avec un masque « maison » en tissu (b). Les gouttes visibles ici (points lumineux), grâce à un éclairage spécifique, ont une taille de l'ordre de la centaine de microns; les gouttes plus petites ne peuvent pas être distinguées individuellement, mais forment un halo visible (indiqué par les flèches). Les gouttelettes (du moins celles visibles à la caméra) sont bien arrêtées par le masque.

(c-d) Visualisation par ombroscopie des écoulements générés lors de la toux, sans masque (c) et avec un masque chirurgical jetable (d). Une partie de l'écoulement passe au travers du masque et est fortement ralentie, tandis qu'une part importante s'écoule par les fuites au niveau du nez, des oreilles et du menton (flèches).

et la pénétration (ou perméance) qui caractérise la quantité de liquide retenue par le filtre en comparant les concentrations en gouttelettes en amont et en aval du filtre. L'évolution se caractérise par trois ou quatre phases [3]. Lors d'une première phase, les gouttelettes se déposent sous forme de perles isolées, et la perte de charge et la pénétration augmentent. Dans une seconde phase, les gouttes s'accumulent et coalescent sur les fibres, jusqu'à former dans une troisième phase des ponts capillaires aux croisements des fibres, puis, enfin, un film liquide couvrant entièrement les fibres. On a alors colmatage du filtre, et la perméance chute tandis que la perte de charge augmente fortement.L'augmentation de la perméance lors des deux premières phases est caractéristique de la filtration de gouttelettes liquides, et est contraire au cas de particules solides où la perméance diminue au fur et à mesure que le filtre se bouche [3]. Les caractéristiques des fibres (diamètre, mouillabilité, flexibilité) ainsi que la structure du filtre (densité et orientation des fibres, nombre de couches) vont conditionner son efficacité de filtrage et la dynamique d'évolution de la perte de charge.

Les modèles actuels restent largement empiriques, et ne permettent pas d'établir de relation directe entre la microstructure et cette dynamique [3]. Néanmoins, plusieurs mécanismes de capture ont été proposés et caractérisés (fig. 2) : (i) l'impact inertiel de gouttelettes qui dévient de l'écoulement et peuvent, de plus, être soumises à des forces extérieures comme la gravité ou des forces électrostatiques (certains masques respiratoires sont en effet traités pour présenter des charges électrostatiques) ; (ii) l'interception directe de gouttes arrivant sur les fibres ; et (iii) la diffusion brownienne qui permet aux plus petites gouttes (de taille inférieure à 0,1 micron) d'être déviées et de rencontrer une fibre (ce troisième mécanisme n'est pas discuté dans cet article).

\section{Impact inertiel}

Dans le cas d'un aérosol fin, dont les gouttelettes sont plus petites que le diamètre des fibres du textile, le mécanisme 


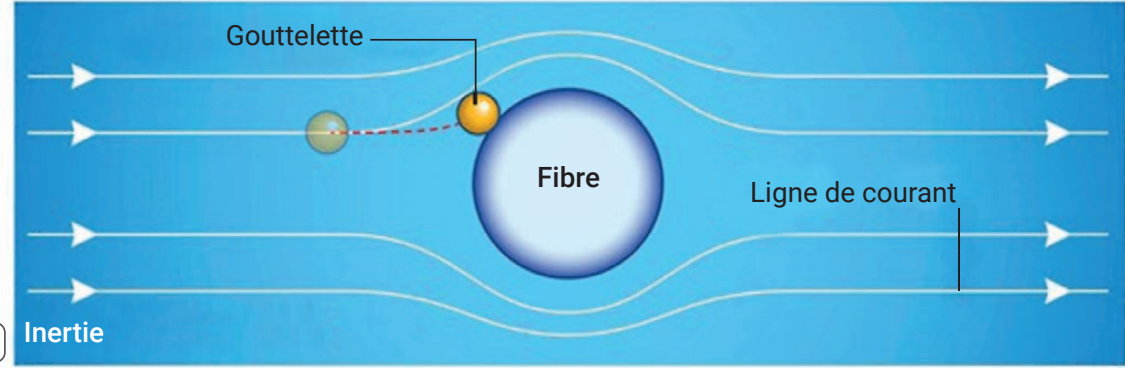

b

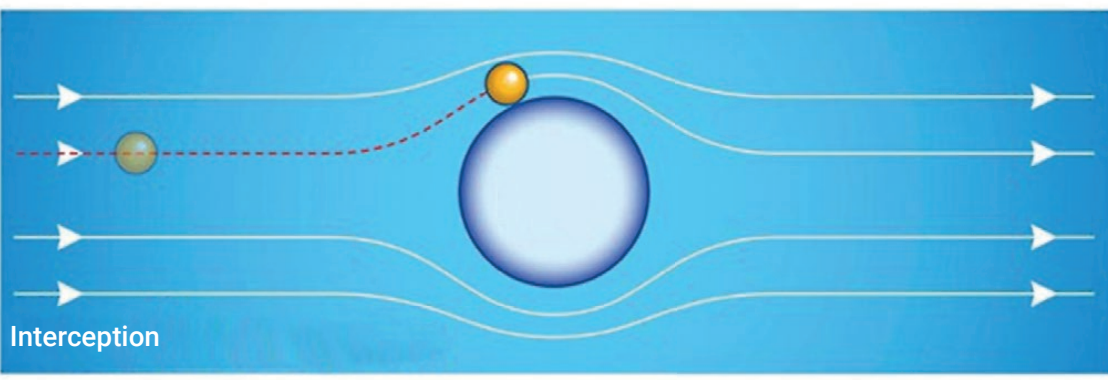

c

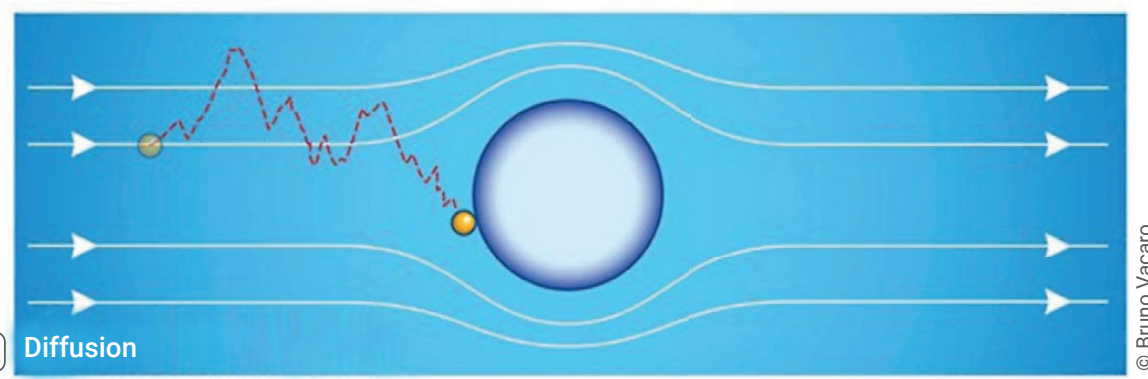

2. Schémas des trois principaux mécanismes de capture d'une gouttelette d'aérosol par une fibre dans un masque respiratoire. Dans le cas où la taille des gouttes est comparable ou supérieure au diamètre des fibres, le mécanisme d'interception directe est différent et décrit dans la référence [5]. (Figure adaptée de J.-M. Courty et É. Kierlik, Pour la Science, n511, mai 2020, pp. 64-66)

\section{$\gg>$}

principal, en l'absence d'effets électrostatiques (qui peuvent être importants pour certains filtres), est l'impact inertiel. En effet, les gouttes contenues dans l'aérosol sont portées par l'écoulement de l'air ; elles vont donc suivre le fluide et éviter les obstacles, sauf si elles possèdent suffisamment d'inertie pour être déviées des lignes de courant et impacter la fibre. Avec des vitesses typiques de l'ordre du mètre par seconde pour l'écoulement d'air, et des tailles de l'ordre du micron pour le rayon des gouttes et de quelques dizaines de microns pour le rayon des fibres, le nombre de Reynolds pour l'écoulement autour des fibres est modéré à élevé (entre 100 et $10000)$, tandis que celui pour l'écoulement autour des gouttes est faible (inférieur à 0,1 ). Ainsi, la trajectoire d'une goutte est donnée par un équilibre entre frottement visqueux et inertie, décrit par le nombre de Stokes (encadré 1), qui compare l'accélération (ou l'inertie) de la goutte autour de l'obstacle
La modification de l'écoulement d'air n'est pas le seul paramètre impactant sur l'efficacité de capture. La présence de liquide sur le réseau de fibres lui-même peut influer sur la capture des gouttes suivantes. À l'aide d'une étude sur des systèmes modèles composés de fibres verticales parallèles, impactées par un aérosol monodisperse de gouttes de 4 microns, nous avons récemment mis en avant le rôle de la distribution spatiale du liquide sur cette collecte par impact inertiel. En effet, la présence de gouttes sur les fibres modifie, non seulement la surface de collecte, mais aussi la forme et la taille des obstacles. Par un phénomène de croissance-coalescence, la distribution de gouttes évolue vers une distribution constante de gouttes uniformément réparties, à l'instar des figures de souffle obtenues lors de la condensation de rosée. En utilisant cette distribution, nous avons ainsi proposé pour la première fois une comparaison quantitative entre des données expérimentales de collecte et le modèle de Langmuir et Blodgett [4].

\section{Interception directe}

Un second mécanisme concerne l'impact de gouttes de taille comparable ou supérieure au diamètre des fibres (c'est le cas notamment des grosses gouttes émises lors des éternuements). Dans ce cas, les gouttes sont ralenties par frottement et par capillarité lorsque la fibre les traverse ; elles se déforment, et peuvent être arrêtées [5]. Ne sont donc capturées que les gouttes arrivant sur les fibres en-dessous d'une vitesse critique de capture, qui dépend du rapport du diamètre des fibres à celui des gouttes, de la physico-chimie du fluide (notamment sa viscosité [6]), ainsi que de la géométrie (alignement, angle d'inclinaison...) et de la flexibilité des fibres [6]. Dans le cas d'une goutte de fluide newtonien peu visqueux, cette vitesse critique dépend du rapport entre l'inertie de la goutte et les forces capillaires, que l'on peut exprimer par un nombre sans dimension, le nombre de Weber (défini dans l'encadré 1). La vitesse critique dépend aussi de la géométrie via le rapport du diamètre de la fibre à la taille de la goutte. Ainsi, à faible nombre de Weber et pour un rapport de tailles important, les effets de tension de surface dominent et peuvent arrêter la goutte, tandis qu'à nombre de Weber élevé et à petit rapport de tailles la goutte ne peut être suffisamment ralentie pour être capturée $[5,6]$. 


\section{Deux mécanismes bien distincts}

Les petites gouttes ne sont capturées qu'au-delà d'une certaine vitesse, lorsque le nombre de Stokes est suffisamment grand, tandis que les grosses gouttes ne seront capturées qu'en dessous d'une vitesse critique, pour un nombre de Weber petit. Si le premier mécanisme est généralement pris en compte dans les modèles de filtration d'aérosols, le second l'est rarement car les aérosols étudiés sont généralement fins. Pourtant, il pourrait être important, car les gouttes émises par un individu ont des tailles comprises entre quelques microns (respiration) et plusieurs centaines de microns (postillons, éternuements).

\section{Comment optimiser l'efficacité de filtration d'un masque}

Finalement, l'efficacité d'un filtre fibreux est donnée en partie par l'efficacité d'une fibre à capturer les gouttelettes, mais dépend aussi de la structure du réseau de fibres, de l'épaisseur du filtre, et de sa perméabilité. En effet, les filtres sont généralement composés de plusieurs couches (trois dans le cas des masques chirurgicaux ou grand public, jusqu'à dix dans d'autres situations) ; si augmenter le nombre de couches augmente l'efficacité en créant plus de possibilités de capture, au-delà d'un certain nombre de couches l'efficacité diminue car la perméabilité chute, et les gouttes contournent le masque en empruntant des
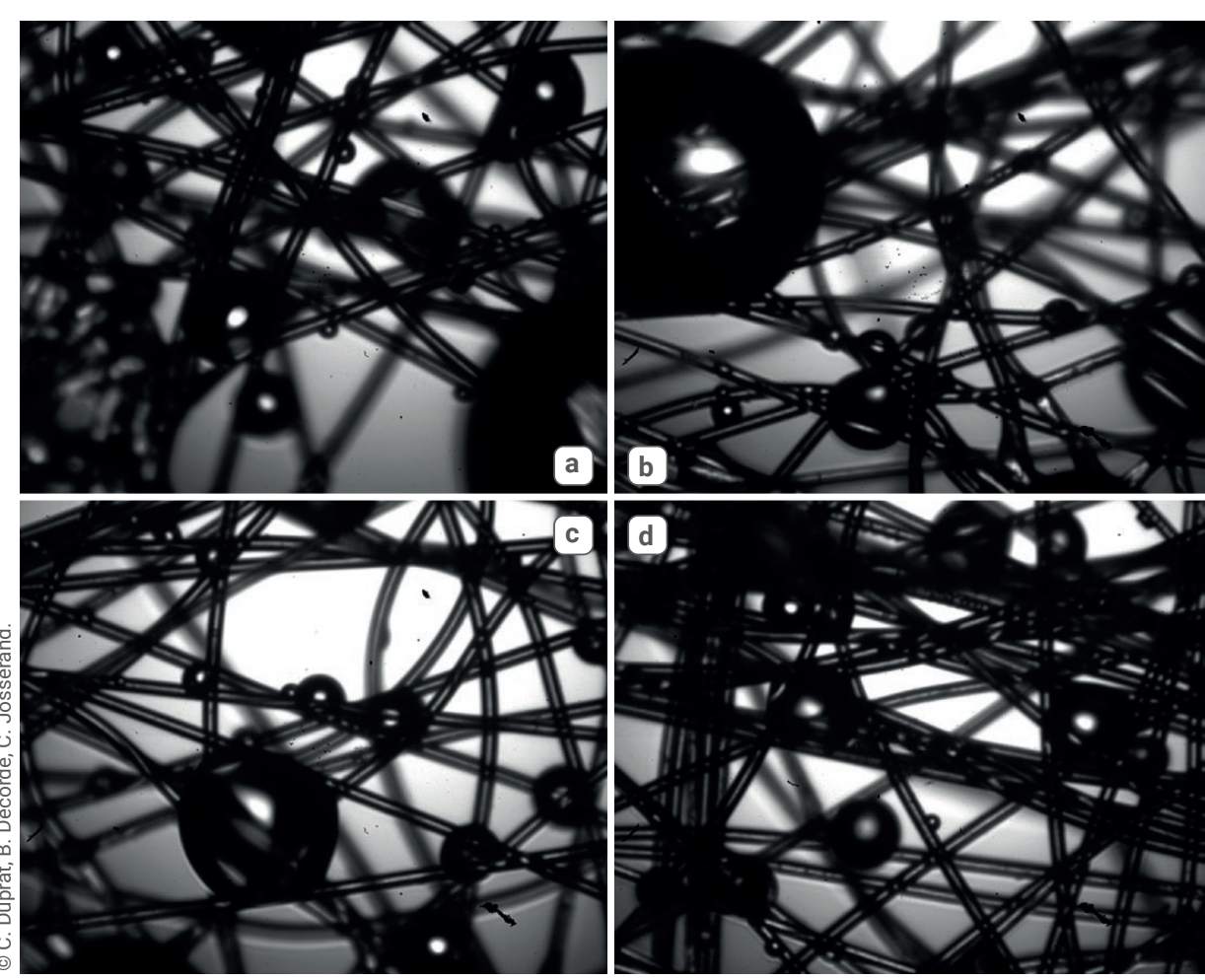

3. Capture de gouttelettes d'aérosol (spray contenant plusieurs tailles de gouttes, de quelques microns à plusieurs centaines de microns) par une couche d'un masque chirurgical. Le diamètre des fibres est de l'ordre de $10 \mu \mathrm{m}$. chemins plus perméables (fuites près du nez ou à l'arrière du masque [7]), ou sont trop ralenties pour être capturées. La proportion d'air expiré qui passe au travers du masque et est donc filtrée, et celle passant par les fuites plus perméables sans être filtrée, dépendent du débit imposé et sont différentes lorsque nous respirons, parlons ou toussons. Il est nécessaire de mieux quantifier ces phénomènes si l'on souhaite créer des masques adaptés.
Nombre de Stokes et nombre de Weber

Encadré 1

Pour une goutte de rayon $r_{d}$, d'un liquide de densité $\rho$ et de tension de surface avec l'air $\gamma$, portée par un écoulement d'air (de viscosité dynamique $\mu$ ) à la vitesse $U$, ou projetée à une vitesse $v$, arrivant sur une fibre de diamètre $d$, on peut définir les nombres sans dimension suivants

- le nombre de Stokes St, qui compare le temps que met une goutte pour être ralentie par les frottements visqueux par rapport au temps que met l'air pour contourner l'obstacle : $S t=4 \rho r_{d}^{2} U /(9 \mu d)$,

- et le nombre de Weber We, qui compare l'accélération de la goutte et les forces capillaires: $W_{\mathrm{e}}=\rho r_{d} v^{2} / \gamma$

On considère des gouttes aqueuses $\left(\rho=1000 \mathrm{~kg} / \mathrm{m}^{3}, \gamma=60 \mathrm{mN} / \mathrm{m}\right)$ impactant des fibres de diamètre $d=10 \mu \mathrm{m}$. Ainsi, des petites gouttes de rayon $r_{d}=3 \mu \mathrm{m}$ portées à $U=1 \mathrm{~m} / \mathrm{s}$ par de l'air chaud $\left(\mu=1,910^{-5} \mathrm{~Pa} . \mathrm{s}\right)$ donnent un nombre de Stokes $S t=20$, et sont donc capturées. Des plus grosses gouttes de rayon $r_{d}=50 \mu \mathrm{m}$ projetées à une vitesse $v=5 \mathrm{~m} / \mathrm{s}$ ont un nombre de Weber $W e=2$, et peuvent être capturées par les fibres.
Bien que prenant en compte ces différents mécanismes, les modèles développés restent largement empiriques. De plus, lorsque les gouttes impactent les fibres, elles doivent y adhérer (fig. 3) ; le mouillage des fibres joue donc un rôle essentiel, bien que peu compris. Le matériau doit être assez mouillant pour que les gouttes y adhèrent, et la force correspondante, caractérisée par l'hystérésis d'angle de contact, assez importante pour éviter qu'elles ne soient réentrainées par l'écoulement. Au sein du filtre, les gouttes vont se déplacer, coalescer, et peuvent saturer le filtre, qui devient alors moins efficace, ou peuvent se détacher et être emmenées par le flux d'air. Ces effets restent aujourd'hui mal caractérisés, et il n'existe pas de relation directe entre la microstructure du filtre et ses propriétés globales de filtration ou de perméabilité. Il est donc nécessaire de mesurer les caractéristiques de chaque masque pour en évaluer l'efficacité, notamment dans le cas de masques artisanaux en tissu, ce qui nécessite de développer des méthodes simples à implémenter [8]. Dans le cas des masques lavables, une norme et un protocole d'essai ont été mis en place pour garantir le maintien des propriétés de filtration et de perméabilité après plusieurs cycles de lavage-séchage (encadré 2). 


\section{Masques lavables}

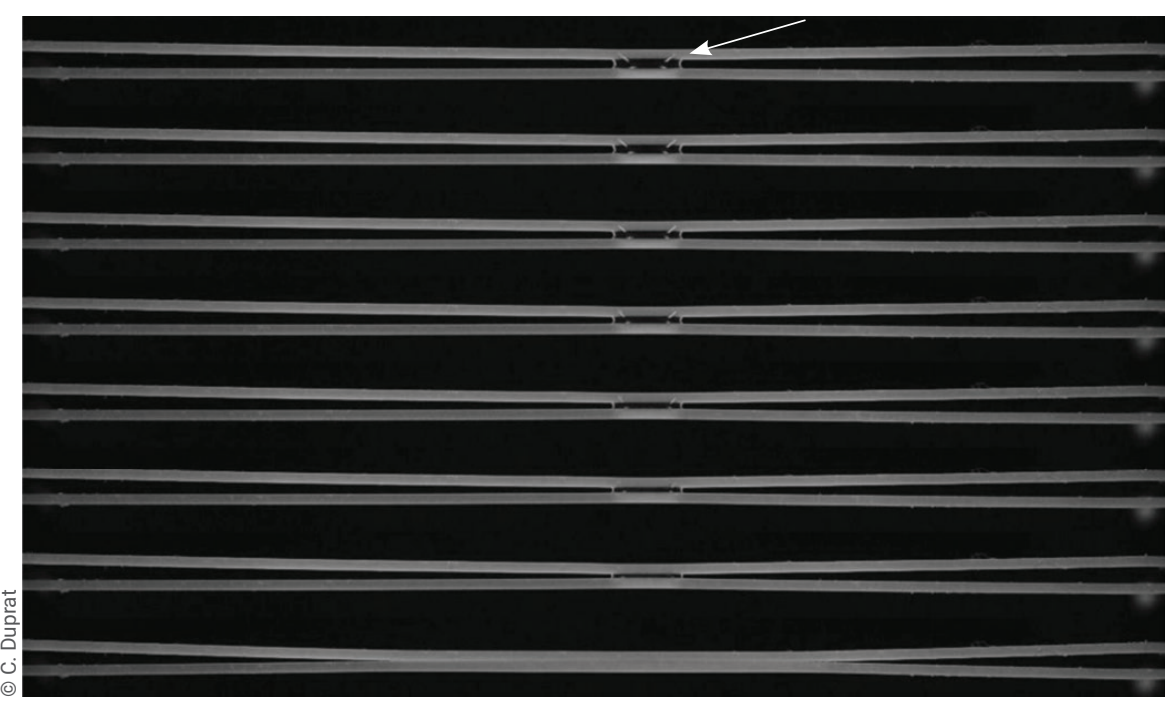

E1. Évaporation d'une goutte (indiquée par une flèche) déposée sur deux fibres flexibles : effondrement des fibres dû aux forces capillaires, en partie à l'origine du rétrécissement observé lors du séchage des textiles.

Comme il n'existe pas de modèle liant microstructure et propriétés globales, il est nécessaire de tester chaque matériau pour chaque application spécifique, et il a fallu mettre en place un protocole pour obtenir une norme pour les masques « maison » lavables, qui offrent une alternative durable aux masques chirurgicaux jetables.

Un protocole d'essai établi par la Direction générale de l'armement (DGA) [9] fixe des critères de perméabilité (respirabilité supérieure à 96 litres. $\mathrm{m}^{-2} \cdot \mathrm{s}^{-1}$ pour une dépression maximale de $100 \mathrm{~Pa}$ ) et d'efficacité de filtration des projections ( $90 \%$ des gouttes de $3 \mu \mathrm{m}$ ), conditions remplies par divers assemblages (masque trois couches tissu coton - non tissé viscose - tissu coton, ou deux couches de non tissé polypropylène ou de tissu coton). L'intérêt de ces masques est qu'ils peuvent être lavés. Néanmoins, la DGA met en garde sur l'effet d'un cycle de lavage-séchage sur les propriétés des masques, qui peuvent alors rétrécir. Ce rétrécissement, témoin d'une déformation de la structure fibreuse, est bien connu, mais finalement peu compris. Il est lié, entre autres, aux forces capillaires qui s'exercent entre les fibres, et dépend de la structure du textile, de la matière, mais aussi du mode de séchage ; ainsi, un textile séché en centrifugeuse/sèche-linge, où les ponts capillaires sont cassés, préserve la structure du textile, tandis qu'un séchage libre sur ligne conduit à un rétrécissement plus fort et une structure plus rigide.

Cet effet peut s'appréhender en regardant un système idéalisé à deux fibres : une goutte s'évaporant entre deux fibres flexibles exerce une force capillaire qui tend à déformer les fibres et à les rapprocher (fig. E1). Cette force augmente lorsque le volume de la goutte diminue, car sa forme évolue [10]. Ainsi, dans une certaine gamme de paramètres, le liquide peut s'étaler spontanément lors du séchage lorsqu'il atteint un volume critique. Pour ce volume, la force exercée est telle que les fibres se trouvent à une distance pour laquelle le liquide préfère s'étaler entre les fibres que rester sous forme de goutte, comme on peut le voir sur la figure E1.

La note de la DGA rapporte que le rétrécissement, obtenu après séchage mécanique, conduit à une amélioration de l'efficacité de filtration (de 92,0\% à 95,4\%), avec, en revanche, une diminution de perméabilité à l'air (en moyenne de $-25 \%$, et jusqu'à - $88 \%$ pour des textiles qui se rétractent beaucoup !). Ces variations sont la signature d'une modification des distances entre fibres, liée en partie aux effondrements entre fibres voisines comme observé sur les modèles à deux fibres, mais aussi sur des membranes fibreuses plus complexes.

\section{$\gg>$}

\section{Conclusion}

Les questions soulevées par les masques sont donc riches, mêlant capillarité, milieux poreux et effets aérodynamiques. Ces systèmes complexes ont été étudiés pour de nombreuses autres applications ; nous avons évoqué la filtration, où les textiles non tissés sont très répandus, mais d'autres usages sont apparus, comme la récupération d'eau douce à partir de brouillard. En parallèle de développements industriels utilisant une approche globale à base de modèles empiriques, se sont développées des études fondamentales sur des systèmes modèles. Il est maintenant essentiel de concilier les mécanismes fondamentaux, à l'échelle de la fibre et du pore, à la réponse globale du textile ; cela ne peut se faire qu'en rapprochant les chercheurs en textiles et les physiciens de la matière molle. Il est aussi essentiel de mieux caractériser la capture et le réentrainement de gouttes par des fibres enchevêtrées, ainsi que les écoulements autour et au travers de textiles flexibles comme les filets à brouillard ou les masques, ce que nous avons démarré, en y associant désormais des médecins.

\section{Références}

1. J.-M. Courty et al., Pour la science $\mathbf{5 1 8}$ (2020) 88-89.

2• I. Langmuir et K. Blodgett, A mathematical Army Airforces Technical Report 5418, Air Technical Service Command, Washington D.C. (1946)

3. R. Mead-Hunter et al., Separation and Purification Technology 133 (2014) 484-506.

4. R. Labbé et C. Duprat, Soft matter 15 (2019) 6946-6951.

5• É. Lorenceau et al., J. Colloid Interface Sci. 279 (2004) 192-197.

6. J. Comtet et al., Soft Matter 12 (2016) 149-156.

7. J.W. Tang et al., Journal of the Royal Society Interface 6 (2009) S727-S736

8. E.P. Fischer et al., Science Advances 6 (2020) eabd3083

9. "Lettre $n^{\circ} 4$ à l'attention des industriels sollicitant DGA. Maîtrise NRBC pour les masques », Direction Générale de l'Armement (3 avril 2020).

10- C. Duprat et H.A. Stone (eds.), "Elastocapillarity", dans Fluid-structure interactions in low-Reynolds-number flows, Royal Society of Chemistry (2015). 